\author{
Robert Kottler ${ }^{1}$ \\ Marko Mank ${ }^{2}$ \\ René Hennig ${ }^{1,3}$ \\ Beate Müller-Werner ${ }^{2}$ \\ Bernd Stahl ${ }^{2}$ \\ Udo Reichl ${ }^{1,4}$ \\ Erdmann Rapp ${ }^{1,3}$ \\ ${ }^{1}$ Max Planck Institute for \\ Dynamics of Complex Technical \\ Systems, Magdeburg, Germany \\ ${ }^{2}$ Danone Research, Centre for \\ Specialised Nutrition, \\ Friedrichsdorf, Germany \\ 3 glyXera GmbH, Magdeburg, \\ Germany \\ ${ }^{4}$ Chair of Bioprocess \\ Engineering, Otto-von-Guericke \\ University, Magdeburg, \\ Germany
}

Received January 15, 2013

Revised April 17, 2013

Accepted April 19, 2013

\section{Development of a high-throughput glycoanalysis method for the characterization of oligosaccharides in human milk utilizing multiplexed capillary gel electrophoresis with laser-induced fluorescence detection}

\begin{abstract}
During the last decade, enormous progress regarding knowledge about composition and properties of human milk (HM) has been made. Besides nutrition, the three macronutrients: proteins, lipids, and carbohydrates combine a large variety of properties and functions. Especially, complex oligosaccharides emerge as important dietary factors during early life with multiple functions. The characterization of these HM oligosaccharides (HMOS) within the total carbohydrate fraction is prerequisite to understand the relationship between milk composition and biological effects. Therefore, extended studies of large donor cohorts and thus, new high-throughput glycoanalytical methods are needed. The developed method comprises sample preparation, as well as analysis of HMOS by multiplexed CGE with LIF detection (XCGE-LIF). Via a respective database the generated "fingerprints" (normalized electropherograms) could be used for structural elucidation of HMOS. The method was tested on HM samples from five different donors, partly sampled as a series of lactation time points. HMOS could be easily identified and quantified. Consequently, secretor and Lewis status of the donors could be determined, milk typing could be performed and quantitative changes could be monitored along lactation time course. The developed XCGE-LIF based "real" high-throughput HMOS analysis method enables qualitative and quantitative high-performance profiling of the total carbohydrate fraction composition of large sets of samples.
\end{abstract}

Correspondence: Dr. Erdmann Rapp, Max Planck Institute fo Dynamics of Complex Technical Systems, Sandtorstraße 1 , Magdeburg, Germany 39106

E-mail: rapp@mpi-magdeburg.mpg.de

Fax: +49-391-6110-535

Abbreviations: 2'-FL, 2'-fucosyllactose; 3-FL, 3-fucosyllactose; aHMOS, total acidic HMOS; APTS, 8-aminopyrene1,3,6-trisulfonic acid; FucT-II and III, fucosyltransferase II and III; GlcNAc, $N$-acetylglucosamine; GPC, gel permeation chromatography; HM, human milk; HMOS, human milk oligosaccharides; HP, high-performance; HPAEC-PAD, high-pH anion exchange chromatography with pulsed amperometric detection; HT, high-throughput; Le, Lewis gene; LNDH II, Lacto- $N$ difuco-hexaose 1-II; LNFP I-III and V, Lacto- $N$-fuco-pentaose I-III and V; LTP, lactation time point; $\mathbf{M S}^{n}$, multistage MS; nHMOS, total neutral HMOS; OS, oligosaccharides; PHP, peak height proportions; PN, peak numbers; RFU, relative fluorescence units; Se, secretor gene; TCF, total carbohydrate fraction; $\mathbf{t}_{\mathbf{m i g}}$, migration time; $\mathbf{t}_{\mathbf{m i g}}$ ', normalized migration time; UF, ultrafiltration; xCGE-LIF, multiplexed CGE with LIF detection

\section{Introduction}

From various points of view, like evolutionary, nutritional, and economical, human milk (HM) is ideal for infant nutrition for the first months of life, and thus it has been recommended by the WHO [1]. Despite the steps that already have been taken regarding knowledge about composition and properties, the functionality of HM in its complexity is still not fully understood [2-6]. Besides water, the three macronutrients of HM are proteins, lipids, and carbohydrates [7-9]. They combine a large variety of properties and functions, which were closely surveyed in several studies. It could be shown that during breastfeeding, antibodies pass from mother to child $[10,11]$, and that $H M$ contains several antiinfective factors such as lactoferrin $[4,6]$ and immunoglobulin $A$, protecting against pathogenic microorganisms [12-14]. All 
this supports the development of the newborns immune system [15-17], e.g. by lowering the risk of infections like otitis media [18] and urinary tract infections [19]. Besides these important factors within the protein fraction of $\mathrm{HM}$, also the total carbohydrate fraction (TCF) is known to contain compounds bearing a variety of complex and important functions beyond simple energy delivery to the infants $[14,20,21]$. The composition of the HM oligosaccharide (HMOS) pool, i.e. the HM-TCF differs, comparing milks from different mothers or from different lactation time points (LTP). In the past, most studies concerning HM and breastfeeding were looking at $\mathrm{HM}$ on its whole and the studies, focusing on the HM-TCF, were analyzing just small donor cohorts (e.g. $[2,6,21-41])$ that can only provide limited information. The HMOS pool mainly consists of lactose $(70-85 \%$ of the HMTCF [31]) and a large variety of neutral and acidic oligosaccharides (OS). Until now, more than 1000 of these HMOS have been detected/identified via different low- and mediumthroughput techniques like chromatography (e.g. paper chromatography [41-45], HPLC [25, 46-48], high-pH anion exchange chromatography with pulsed amperometric detection (HPAEC-PAD) [26, 49-54] or hydrophilic interaction chromatography [55]), MS [56] (e.g. using infrared laser desorption MS [57], fast atom bombardment ionization com. bined with MS/MS, [58] MALDI-MS/MS [23, 24, 59-65], (nano) ESI (multistage) MS ((nano)ESI-MS $\left.{ }^{(n)}\right)[66-69]$ or using a high-resolving Fourier transform ion cyclotron resonance MS [70]), LC coupled online with (multistage) MS $\left(\right.$ LC-MS $\left.{ }^{(n)}\right)$ [71-79], NMR spectroscopy $[80,81]$ or single CE with LIF detection $[82,83]$, sometimes additionally coupled online with MS [84-86]. But, as the characterization of HMOS within the HM-TCF is prerequisite to understand the complex relationship between their structures and their biological effects [28], there is a need for high-performance (HP) analytical methods that combine high sensitivity, high selectivity, and high resolution with robustness, reliability, and reproducibility in a high-throughput (HT) manner [83].

Consequently, our work is focusing on the development and establishment of an HP glycoanalytical technique, based on multiplexed CGE with LIF detection (XCGE-LIF) that allows in-depth characterization of the HM-TCF composition in large numbers of individual (human) milk samples. The presented method comprises sample preparation, as well as xCGE-LIF analysis of HMOS, both ready for "real" HT. First neat HMOS were isolated out of the HM-TCF. These neat compounds (HMOS standards) were then used for method development and reproducibility testing. Following on that, we initiated a database (containing said HMOS standards and their normalized migration times $\left.\left(\mathrm{t}_{\mathrm{mig}}{ }^{\prime}\right)\right\}$, for a later on fast and easy identification of HMOS beyond peaks of "fingerprints" (normalized electropherograms) generated from real HM samples. To approve the methods capability for monitoring TCF compositions of larger cohorts of individual HM samples in future, we tested the developed method with the HM-TCF of four different donors (representing the four known HM types, according to Thurl et al. [87]), as well as a series of eight HM samples of one donor from different
Table 1. Samples and lactation time points

\begin{tabular}{|c|c|c|c|c|c|c|c|c|c|}
\hline $\begin{array}{l}\text { LTP } \\
\text { (days postpartum) }\end{array}$ & $\begin{array}{l}1 \\
(1-3)\end{array}$ & $\begin{array}{l}2 \\
(4)\end{array}$ & $\begin{array}{l}3 \\
(8)\end{array}$ & $\begin{array}{l}4 \\
(15)\end{array}$ & $\begin{array}{l}5 \\
(22)\end{array}$ & $\begin{array}{l}6 \\
(30)\end{array}$ & $\begin{array}{l}7 \\
(60)\end{array}$ & $\begin{array}{l}8 \\
(90)\end{array}$ & Pool \\
\hline $\mathrm{HMI}$ & & & & & & & & & Yes \\
\hline HM II & & & & & & & & & Yes \\
\hline HM III & & & & & & & & & Yes \\
\hline HMIV & & & & & & & & & Yes \\
\hline HMV & & & & & & & & & Yes \\
\hline HM VI & $x$ & $x$ & $x$ & $x$ & $x$ & $x$ & $x$ & $x$ & No \\
\hline
\end{tabular}

time-points along the lactation time course. It turned out that this xCGE-LIF based "real" HT technique represents a valuable alternative to existing glycoanalysis tools that are more time, lab space, and manpower consuming. This technique will enable unequivocal qualitative and quantitative HP profiling of the HM-TCF (i.e. the HMOS pool) composition of large cohorts.

\section{Materials and methods}

\subsection{Samples}

All HM samples (Table 1) were provided from healthy volunteers; Caucasian women living in the regions of Dresden (Germany) and Frankfurt/Main (Germany) (previously used by Thurl et al. [27,87], see also for further sample details). The HM samples were immediately frozen after delivery and stored at $-20^{\circ} \mathrm{C}$ until analysis. Pooled sample HM I was used for the isolation of neat HMOS. Samples HM II-V were used for HM type determination of their donors. The series of eight HM samples of one donor from different timepoints (HM VI) was taken to demonstrate the methods capability for monitoring HM-TCF composition along the lactation time course.

\subsection{Sample preparation and analysis workflow for HMOS profiling via XCGE-LIF}

Figure 1 shows the HMOS sample preparation and analysis workflow. Briefly, all samples were heated for $30 \mathrm{~min}$ at $70^{\circ} \mathrm{C}$ according to [88] (pasteurization) to inactivate pathogens and to prevent degradation of HM. In the following, proteins and lipids were separated from carbohydrates either via ultrafiltration (UF) (Fig. 1, left branch) or via a two-step purification, with first skimming via centrifugation and second deproteinization via UF (Fig. 1, right branch), resulting in the isolated HM-TCF. Subsequently, the HM-TCF could be analyzed directly or optionally further fractionated by chromatographic steps (Fig. 1, right branch) to generate HMOS fractions and neat HMOS thereof. To unravel and detect the extracted HMOS using XCGE-LIF, they were fluorescently labeled with 8-aminopyrene-1,3,6-trisulfonic acid (APTS). Sulfonated aromatic amines, like 8-aminonaphthalene-1,3, disulfonic acid, 8-aminonaphthalene-1,3,6-trisulfonic acid 


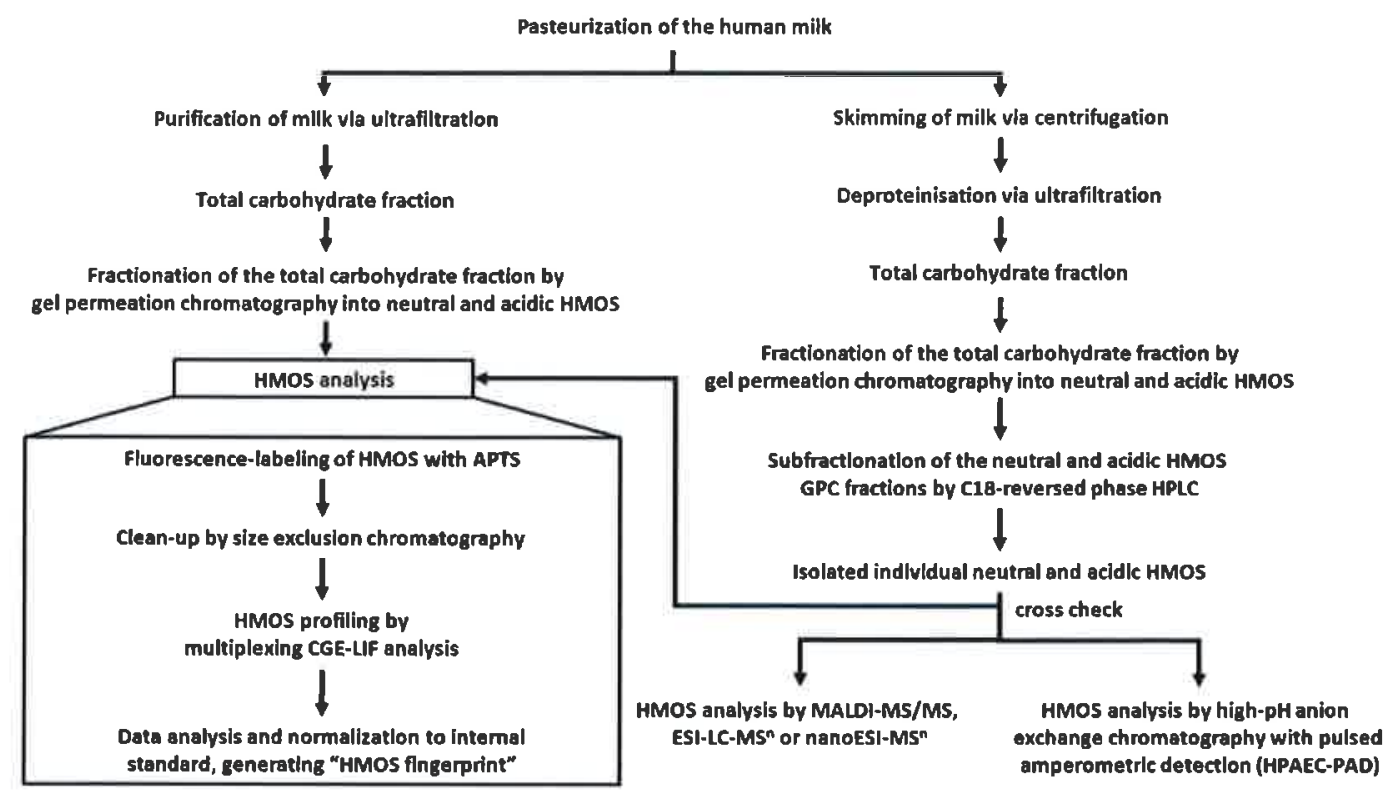

Figure 1. Workflow of XCGE-LIF based OS analysis from HM (left branch) and isolation/verification of the HMOS standards (right branch).

(ANTS), and APTS are common fluorescent derivatives widely used in the field of electrokinetic separation techniques [89-91]. Due to the utilization of an argon-ion laser inside the $x$ CGE-LIF system with an excitation wavelength of $488 \mathrm{~nm}$ and a band filter at $524 \mathrm{~nm}$, APTS (with a $\lambda_{\text {exc_max }}$ of $455 \mathrm{~nm}$ and a $\lambda_{\text {ent_max }}$ of $512 \mathrm{~nm}$ ) was chosen as fluorescent tag, perfectly fitting the requirements. For postlabeling purification, the mixture of excess APTS and APTS-labeled HMOS was fractionated via SEC according to Schwarzer et al. [92]. Following this postlabeling and clean-up step, the HMOS containing fractions were identified, pooled, and analyzed using xCGE-LIF according to [92-94].

\subsubsection{Sample preparation of the pooled HM samples HM II-V}

Lipid and protein contents were reduced by centrifugation and UF at $2000 \times \mathrm{g}$ and $6^{\circ} \mathrm{C}$ using the Centrifree micropartition system (centrifugal filter devices, $30 \mathrm{MWCO}$, regenerated cellulose, $1 \mathrm{~mL}$, 4104, Amicon, Germany) using an Avanti J-26 XP centrifuge (with JS-7.5 rotor, Beckman Coulter, USA) according to [88]. By gel permeation chromatography (GPC), the remaining aqueous UF-permeate (crude TCF) was separated into monosaccharides, lactose, the total acidic HMOS fraction (aHMOS) and the total neutral HMOS fraction (nHMOS), as described in detail by Thurl et al. [88]. Briefly, the separations were performed on a semipreparative LC system (pump P-500 (GE, Netherlands); 3-canal-degaser (ERC, Germany); two serially connected, thermo jacket $(4.0 \times 110 \mathrm{~cm}$ ) glass columns (Kronlab, Germany), packed with Toyopearl HW 40 (S) (14681, Tosoh Bioscience, Japan); column temperature: $60^{\circ} \mathrm{C}$, via col- umn heating bath RK 8 KS (Lauda, Germany) connected to thermo jacket of the glass columns; refractive index (RI) detector LKB Bromma 2142 differential refractometer (GE); fractionator (SuperFrac, GE); recorder SE 120 (BBC Goerz Metrawatt, Germany), and software AI-450 (Version 3.1, Dionex, USA)). The eluent was MilliQ ${ }^{T M}$ water $+2 \% \mathrm{v} / \mathrm{v}$ 2-propanol (278 475, Sigma Aldrich, Germany); packing flow rate was $1.7 \mathrm{~mL} / \mathrm{min}$; separation flow rate was $1.3 \mathrm{~mL} / \mathrm{min}$; injected sample weight/volume was $1 \mathrm{~g} / 5 \mathrm{~mL}$. The aHMOS and nHMOS fractions were dried by lyophilization using the Alpha I-12 lyophilisator (Christ, Germany) with RC-5 pump (VacuuBrand, Germany) (temperature: $-20^{\circ} \mathrm{C}$ and pressure: $20 \mathrm{mbar}(\Delta \mathrm{p} 3 \mathrm{mbar})$ ) and then redissolved in $1 \mathrm{~mL}$ or $0.5 \mathrm{~mL}$ of MilliQ ${ }^{\mathrm{TM}}$ water, respectively.

\subsubsection{Sample preparation of HM samples HM VI (lactation time course series)}

Here the starting point was $1 \mathrm{~mL}$ of $\mathrm{HM}$ already mixed with $0.1 \mathrm{~mL}$ of an aqueous solution containing stachyose $(400 \mathrm{mg} / 100 \mathrm{~mL})$ and galacturonic acid $(80 \mathrm{mg} / 100 \mathrm{~mL}$ ). which were added as internal standards in previous studies $[26,27]$. Again first, lipid and protein contents were reduced, as described above. The remaining crude HM-TCF was fractionated by GPC, dried, and dissolved, as described above.

\subsubsection{Sample preparation for generation of HMOS standards from pooled HM sample HM I}

To generate HMOS standards and to build-up a HMOS library and a database, neat HMOS were isolated from 


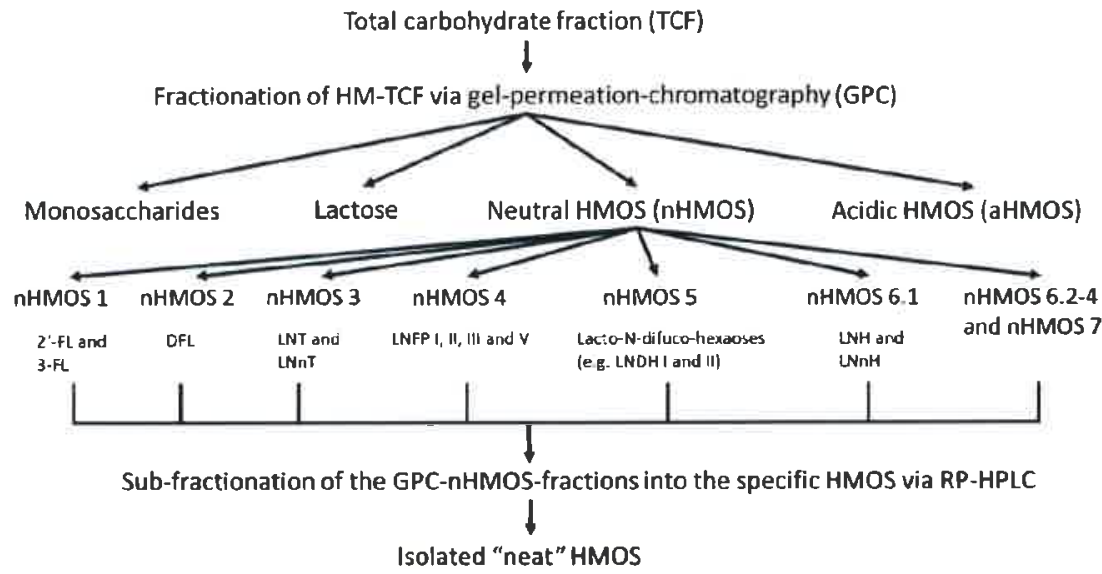

Figure 2. Scheme for fractionation of HM-TCF into monosaccharides, lactose, the neutral and the acidic HMOS fractions, followed by further subfractionation into neat HMOS (HMOS standards for XCGE-LIF database).
HM I (pooled HM). Again first, the lipid and protein contents were reduced. Therefore, a two-step method was used: First, the HM was skimmed using an Avanti J-26 XP centrifuge $\left(15 \mathrm{~min}, 5000 \mathrm{rpm}\right.$ and $4^{\circ} \mathrm{C}$, with JS-7.5 rotor, Beckman Coulter). The remaining aqueous solution was filtered through glass wool (18421, Sigma Aldrich) to totally skim the cream. In a second step, the filtered aqueous solution was deproteinized via UF (reservoirs: $150 \mathrm{~L}$; feed flow rate: $2.4 \mathrm{~m}^{3} / \mathrm{h}$ with max. 8.5 bar; circulation flow rate: $20 \mathrm{~m}^{3} / \mathrm{h}$ ). For this purpose a $5 \mathrm{~m}^{2}$ polyethersulfone/polysulfone plate module membrane with a molecular cut off of $30 \mathrm{kDa}$ (Lenntech, Netherlands) was used. Afterwards, the remaining filtrate was spray dried. The spray dried sample was dis. solved $(1 \mathrm{~g} / 5 \mathrm{~mL})$ in MilliQ ${ }^{\mathrm{TM}}$ water. By GPC (see Fig. 1 , right branch) and similar as described above, this crude HM-TCF solution again was separated into monosaccharides, lactose, aHMOS, and nHMOS fractions. Briefly, the separations were performed on a semipreparative LC system (pump LKB 2150 (GE); double jacketed $(1.6 \times 90 \mathrm{~cm})$ glass column (Kronlab) packed with TSK HW-40(S) (Merck, Germany); column temperature: $60^{\circ} \mathrm{C}$, via column heating bath RK 8 KS (Lauda); refractive index-detector LKB Bromma 2142 differential refractometer (GE); fractionator LKB Frac100 (GE)). The eluent was MilliQ ${ }^{\text {TM }}$ water $+0.02 \%$ v/w sodium azide $(71289$, Sigma Aldrich); packing flow rate was $1.5 \mathrm{~mL} / \mathrm{min}$; separa. tion flow rate was $1.0 \mathrm{~mL} / \mathrm{min}$; injected sample volume was $100 \mu \mathrm{L}$. But, this time the GPC nHMOS and aHMOS fractions were collected as subfractions (nHMOS 1.7 and aHMOS 1-6) (Fig. 2). Subsequently, the individual subfractions were vacuum dried using a vacuum centrifuge RVC $2-25$ with cooling trap CT $02-50$ at $-50^{\circ} \mathrm{C}$ (Christ) and vacuum pump MZ25 (VacuuBrand) and were stored at $-20^{\circ} \mathrm{C}$ until further processing.

According to Stahl et al. [60], for the purpose of HMOS isolation, each of these nHMOS and aHMOS subfractions were resolved in MilliQ ${ }^{\mathrm{TM}}$ water, at a concentration of $50 \mathrm{~g} / \mathrm{L}$ and individually reinjected and further separated and fractionated (see also Fig. 2) on a semipreparative RP-HPLC system, (two pumps: model 215 with $5 \mathrm{~mL}$ stainless-steel pump heads (Varian, Germany); two serially connected (8.0 $\times 250 \mathrm{~mm}$ ) columns with a $30 \mathrm{~mm}$ precolumn, packed with NUCLEOSIL ${ }^{(B)} 100-5 \mathrm{C}_{18} / 5 \mu \mathrm{m} / 100$ A (MACHEREYNAGEL, Germany); UV-detector UV1 (Dynamax, USA) (195 nm); fractionator model 704 (Varian), fractionation intervals: $10-30 \mathrm{~s}$ ). Eluent: MilliQ ${ }^{\mathrm{TM}}$ water; flow rate of $0.8-$ $1.2 \mathrm{~mL} / \mathrm{min}$ (at room temperature): injected sample volume was $25 \mu \mathrm{L}$; ethanol $(99.8 \%, 34852$, Sigma Aldrich) was used for column regeneration. To isolate the compounds, the RPHPLC flow rates and fractionation intervals had to be optimized for each GPC fraction. If required, the RP chromatographic step was repeated for the collected subfractions until just one single HMOS structure was identified within them (data not shown). HMOS identification and purity checking was done either via MALDI-MS/MS, according to [60,61], nanoESI-MS" (where $\mathrm{MS}^{\mathrm{n}}$ is multistage MS) according to Pfenniger et al. $[66,69]$, ESI-LC-MS ${ }^{n}$, according to Thomsson et al. [74], and/or HPAEC-PAD, according to Thurl et al. [26].

Differing from $[60,61]$ a MALDI-TOF/TOF 4800 instrument (ABSciex, USA) was used and operated in positive reflector ion-mode utilizing dried droplet preparation and DHBS-MALDI-Matrix (20 g/L in 5\% ACN (34 967, Sigma Aldrich) with $0.1 \%$ v/v TFA (>99\%, 40967. Sigma Aldrich)) as matrix, according to Karas et al. [95]. In contrast to Thomsson et al. 174] for ESI-LC-MS" analysis of native HMOS, we employed an LTQ Velos linear ion trap instrument (Thermo Scientific, USA) online coupled to an Ultimate 3000 HPLC system (both: ThermoScientific) operated with a porous graphitized carbon (PGC) column $(1.0 \times 100 \mathrm{~mm}$, packing: Hypercarb, $3 \mu \mathrm{m}$ particle size, ThermoScientific) at room temperature and a flow rate of $20 \mu \mathrm{L} / \mathrm{min}$. Furthermore, a 170 min MilliQ ${ }^{\mathrm{rM}}$ water-ethanol gradient was used instead of the original $\mathrm{H}_{2} \mathrm{O}-\mathrm{ACN}$ gradient. Eluents were $\mathrm{MilliQ}^{\mathrm{TM}}$ water (= eluent A) and ethanol (= eluent B) $(99.8 \%$, 34 852, Sigma Aldrich), both contained $0.1 \%$ formic acid v/v (98\%, 94 318, Sigma Aldrich). Gradient profile was as follows: 0-11 min 100\% A, 11-75 min 100-70\% A, 75-85 min 70$0 \%$ A, 85-125 min 0\% A, 125-130 min 0-100\% A, 130170 min $100 \%$ A. IEC-based HMOS analyses were performed according to [26] on an HPAEC-PAD system (DX-300 BIOLC-system (Dionex); AS3500 autosampler (Spectra Physics, USA): Carbo-Pac PA-100 $(4.0 \times 250 \mathrm{~mm})$ column with 
CarboPac PA-100 (4.0 × $50 \mathrm{~mm})$ precolumn (both: Dionex): pulsed electrochemical detector PED-2 (Dionex)). Eluents were $\mathrm{MilliQ}^{\mathrm{TM}}$ water and aqueous solutions of sodium hydroxide (NaOH, 306576 , Sigma Aldrich) and sodium acetate (NaOAC, S3272, Sigma Aldrich). They were used to generate the following gradient: $0-20 \mathrm{~min}, 30 \mathrm{mM} \mathrm{NaOH}$; 20-34 min, 30-100 mM NaOH; 34-48 min $100 \mathrm{mM} \mathrm{NaOH} /$ 0-28 mM NaOAc; 48-55 min, $100 \mathrm{mM} \mathrm{NaOH} / 28-200 \mathrm{mM}$ $\mathrm{NaOAC}$; 55-60 min $100 \mathrm{mM} \mathrm{NaOH} / 200 \mathrm{mM} \mathrm{NaOAc}$; flow rate was $1.0 \mathrm{~mL} / \mathrm{min}$ (at room temperature).

With this procedure, at least $1 \mathrm{mg}$ of each of the 17 neat HMOS compounds was sampled pooling the respective fractions from several semipreparative RP-chromatographic runs.

\subsubsection{Fluorescent labeling, postlabeling clean-up, and XCGE-LIF based HMOS analysis}

After protein and lipid content of HM were reduced via UF, the TCF was analyzed as shown in Fig. 1 (left branch of the scheme). Therefore, the HMOS were fluorescently labeled according to the method of Callewaert et al. [96] and modified by Schwarzer et al. [92] with APTS $(>96.0 \%$, A7222, SigmaAldrich) by reductive amination. Briefly, $2 \mu \mathrm{L} 20 \mathrm{mM}$ APTS and $2 \mu \mathrm{L} 1 \mathrm{M}$ sodium cyanoborohydride $\left(\mathrm{NaCNBH}_{3}\right)$ (reagent grade, 156 159. Sigma Aldrich) both in 15\% v/v acetic acid (99.7\%, A3701, Applichem, Germany) in MilliQ ${ }^{T M}$ water were added to $2 \mu \mathrm{L}$ of the TCF. Derivatization was allowed for $18 \mathrm{~h}$ at $37^{\circ} \mathrm{C}$. Afterwards, the excess of APTS (not reacted) and salt were removed using SEC. Therefore, MultiScreen Deep Well Solvinert Filter Plates (MDRL N04, low-binding hydrophilic PTFE, Millipore, USA) were packed with Toyopearl HW-40(F) (19 808, Tosoh Bioscience, Japan). Before packing, the slurry was washed six times with $\mathrm{MilliQ}^{\mathrm{TM}}$ water in $50 \mathrm{~mL}$ centrifuge tubes. Then, deep well filter plates were packed with $2 \mathrm{~mL}$ washed slurry and washed again with three column volumes MilliQ ${ }^{\mathrm{TM}}$ water. After sample application, HMOS were eluted with $\mathrm{Milli}^{\mathrm{TM}}$ water by centrifugation $(50 \times \mathrm{g} / \mathrm{step})$. Up to 25 fractions were collected per well (each in one 96-well plate (V-bottom Polystyrene, 9292.1, Roth, Germany)) and screened for HMOS content by analyzing the fractions with a 4-capillary DNA-sequencer "ABI PRISM 3100-Avant Genetic Analyzer" (Applied Biosystems, USA). Afterwards, HMOS containing fractions were pooled. HMOS "fingerprints" were obtained by analyzing the HMOS pools via XCGE-LIF according to Schwarzer et al. [92]. Each of the four parallel capillaries had an effective capillary length of $50 \mathrm{~cm}$. Undiluted POP $-6^{\mathrm{TM}}$ polymer (4 316357, Applied Biosystems) was used as separation matrix. Samples were diluted 1:10 in $\mathrm{HiDi}^{\mathrm{TM}}$-Formamide (4 311320 . Applied Biosystems) and injected for $5 \mathrm{~s}$ at $15 \mathrm{kV}$. Separation was performed for $130 \mathrm{~min}$ at $15 \mathrm{kV}$ and $30^{\circ} \mathrm{C}$. According to Schwarzer et al. [92] internal standard was added to the samples after diluting with $\mathrm{HiDi}^{\mathrm{TM}}$-Formamide. Based on this standard, a migration time normalization was performed using the Matlab ${ }^{\circledR}$ based program (gl/Xtool ${ }^{T M}$ )
[97], that enabled automated data processing and analysis. Doing so, the migration time $t_{\text {mig }}$ in (data points) was automatically normalized to $t_{\text {mig }}$ ' with normalized migration time units (MTU').

\subsubsection{Reproducibility testing and HMOS database built-up}

For reproducibility testing and to initiate a respective HMOS database, normalized xCGE-LIF data resulting from repeated analysis of the 17 isolated and identified neutral HMOS standards (see above) were compiled. Over a timespan of six months, these standards were measured, at least ten times each. Based on these repeated measurements, the mean normalized migration times and the resulting RSDs were calculated (Table 2).

\subsubsection{Peak identification via migration time matching with HMOS database entries}

Based on the results of the reproducibility testing ( $R S D<1 \%$, for all isolated and tested HMOS standards), the new HMOS database (correlating HMOS structures with their respective $\mathrm{t}_{\text {inig }}{ }^{\prime}$ (Table 2)) was used to assign HMOS to xCGE-LIF peaks. Peaks with the same $t_{\text {mig }}$ most likely represent the same HMOS. For selected peaks/HMOS, this was cross-checked via spiking experiments (as described below/data not shown).

\subsubsection{Peak identification via spiking experiments}

For spiking experiments, the isolated and labeled HMOS standards were used. The respective HMOS standard was added to the sample, the HiDi ${ }^{\mathrm{TM}}$-Formamide volume to be added was accordingly reduced. Thus, end-volume and concentration of the sample were the same and the run was conducted as described above (data not shown).

\subsubsection{Quantitative comparison of HMOS fingerprints at different lactation time points via the relative peak height proportions (PHP)}

HMOS concentration changes during lactation were analyzed by calculating relative PHP (as published previously for $\mathrm{N}$-glycosylation patterns $[92,98-103])$. Briefly, the same set of 18 peaks was picked in all HMOS fingerprints of the samples from the ITP series (peak picking criteria: peaks $>200$ relative fluorescence units (RFU) or already known peaks). To determine the relative PHP of the peaks, the peak heights (intensities in RFU) of all picked peaks were summed up to the total peak height. Then the relative PHP of each picked peak could be determined in percent of the total peak height, i.e. the peak height of each picked peak was normalized to the sum of the peak heights 
Table 2. Initiated HMOS/XCGE-LIF tmig' database

\begin{tabular}{|c|c|c|c|}
\hline \multirow[t]{2}{*}{ Name } & \multicolumn{2}{|c|}{ HMOS } & \multirow{2}{*}{$\begin{array}{l}\text { Peak number } \\
\text { (PN) }\end{array}$} \\
\hline & Structure & 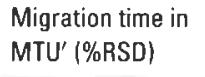 & \\
\hline Lactose & 0,0 & $22.07(3.1)$ & 1 \\
\hline 3-FL & 0,1 & $46.50(0.2)$ & 2 \\
\hline $2^{\prime}-\mathrm{FL}$ & $-: 0,0$ & $47.92(0.8)$ & 3 \\
\hline DFL & $0.0,9$ & $64.87(0.3)$ & 4 \\
\hline LNnT & $0,1=0,0$ & $87.62(0.9)$ & 5 \\
\hline LNT & $0,1,{ }^{n}, O_{1}, 0$ & $90.40(0.7)$ & 6 \\
\hline LNFP II & $\mathrm{O}_{5}$ & $109.88(0.5)$ & 7 \\
\hline LNFP V & $0,0,0,0$ & $111.67(0.4)$ & 8 \\
\hline LNFP I & 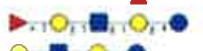 & $113.03(0.6)$ & 9 \\
\hline LNFP III & Ori & $115.09(0.3)$ & 10 \\
\hline $\mathrm{LNnH}$ & 0.1 每 & $121.25(0.6)$ & 11 \\
\hline LNH & $\mathrm{O}, \mathrm{x}$ & $121.73(0.6)$ & 12 \\
\hline LNDH I & $\mathrm{DO}_{\mathrm{O}, \mathrm{O}}$ & $126.63(0.2)$ & 13 \\
\hline LNDH II & $\mathrm{O}_{1}$ & $128.68(0.2)$ & 14 \\
\hline 3-F-LNH & 0,7 & $140.47(0.6)$ & 15 \\
\hline $2^{\prime}-\mathrm{F}-\mathrm{LNH}$ & & $141.72(0.6)$ & 16 \\
\hline 2,3-DF-LNH & 0, i & $161.24(0.5)$ & 17 \\
\hline
\end{tabular}

Mean and $R S D$ values of $t_{\text {mig }}{ }^{\prime}$ were calculated from $\geq 10$ repeated measurements over 6 months. Schematic HMOS structures were generated with GlycoWorkbench (EuroCarbDB) [132], according to CFG nomenclature; $N$-acetylglucosamine ( $\mathrm{Q}$, galactose (1) glucose (d), and fucose (ㅅ. 2'-FL, 2'-fucosyllactose; 3-FL, 3-fucosyllactose; $2^{\prime}-\mathrm{F}-\mathrm{LNH}$ : $2^{\prime}$-fucosyl-lacto- $N$-hexaose; 3-F-LNH: 3-fucosyl-Lacto- $N$-hexaose; DFL: Difucosyllactose; LNDH I-HI, Lacto- $N$-difuco-hexaose I-II; 2,3-DF-LNH: 2,3-difucosyl-Lacto- $N$-hexaose; LNFP I-III and V. Lacto- $N$-fuco-pentaose I-III and V; LNH: Lacto- $N$-hexaose; LNnH: Lacto- $N$-neo-hexaose; LNT: Lacto- $N$-tetraose; LNnT: Lacto$N$-neo-tetraose
Table 3. Classification of HM types based on the presence and absence of specific HMOS fucosylation

\begin{tabular}{|c|c|c|c|}
\hline \multirow[t]{2}{*}{ Milk type } & \multicolumn{2}{|c|}{ Specific fucosylation } & \multirow[t]{2}{*}{ Relative distribution $(\%)^{\text {a }}$} \\
\hline & $\alpha 1-2(S e)$ & $\alpha 1-4(L e)$ & \\
\hline 1 & + & + & 69 \\
\hline II & - & + & 20 \\
\hline III & + & - & 10 \\
\hline IV & - & - & 1 \\
\hline
\end{tabular}

Se-secretor gene encodes the fucosyltransferase II that catalyzes OS epitopes with an $\alpha 1-2$ fucosylation at end terminal galactose; Le-Lewis gene encodes the fucosyltransferase III that catalyzes $O S$ epitopes with an $\alpha$ 1-4 fucosylation at $\mathrm{N}$-acetylglucosamine a) [87]

of all picked peaks. Despite the fact, that peaks with the same $t_{\text {mig }}$ ' most likely represent the same HMOS and thus, easily could be assigned via $t_{\text {mig }}$ ' matching with database entries again, all the respective peaks were verified via cross-checking with spiking experiments (data not shown).

\section{Results}

\subsection{Reproducibility testing and initialization of an xCGE-LIF based HMOS database and library}

In order to build up an HMOS-database, first, the reproducibility of the normalized migration times of the respective HMOS standards had to be demonstrated. Therefore, a reproducibility study was performed, periodically $(N \geq 10)$ measuring these HMOS standards over a timespan of six months. As described in Section 2 (Fig. 1), the standards were isolated from HM via RP-HPLC after GPC-fractionation of total HM-TFC, identified and labeled. In addition, the purity of the standards has been cross-checked via HPAEC-PAD, MALDI-MS/MS, nanoESI-MS ${ }^{n}$, and/or ESI-LC-MS ${ }^{n}$ - and their structures were elucidated by these orthogonal methods (described in Section 2 - data not shown). Evaluating the repeated measurements of the isolated APTS-labeled HMOS standards (data not shown), the needed high reproducibility of $t_{\text {mig }}$ ' was approved (long-term RSD $<1 \%$ ). Doing so, an initial HMOS database could be built up, including a first set of 17 HMOS (Table 2).

\subsection{Approval of XCGE-LIF based method regarding qualitative and quantitative analysis of real HMOS samples}

Method validation and feasibility testing was done via two series of experiments. First, exemplary HMOS fingerprints of four different donors were recorded via xCGE-LIF with respect to HM typing (Table 3 ) and structural elucidation of HMOS in complex mixtures. The generated individual fingerprints are shown in Fig. 3, i.e. the qualitative HMOS 

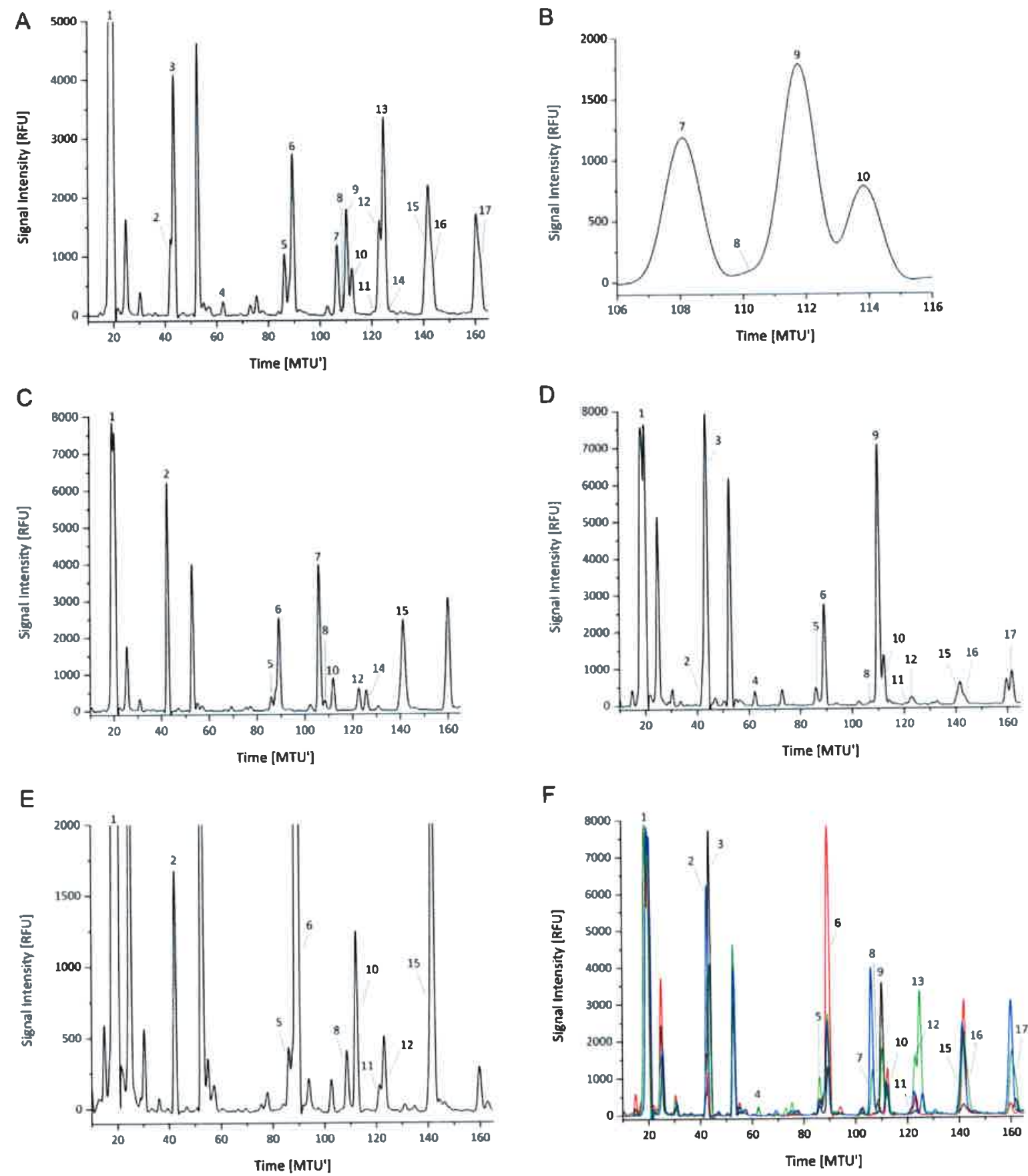

Figure 3. HMOS fingerprints (normalized electropherograms) representing the four HM types. Fingerprints (relative fluorescence units [RFUI plotted over normalized migration time units [MTU']) of the HM-TCF generated via xCGE-LIF. Peak numbers (PN 1-17) according to Table 2. Fingerprints of HM samples (Table 1): (A) HM II; (B) zoom-in (106-116 MTU') of (A); (C) HM III; (D) HM IV; and (E) HM Vrepresenting HM type I, II, III, and IV, respectively (according to Table 3) and (F) Overlay of the HMOS fingerprints (green: HM type I (HM II); blue: HM type II (HM III); black: HM type III (HM IV) and red: HM type IV (HM V).

composition of different HM was investigated. Second, HMOS fingerprints of different LTP of one donor along the lactation time course were measured in three technical replicates to demonstrate methods feasibility regarding relative quantitation. The series of generated individual fingerprints representing the lactation time course is shown in Fig. 4A. According to Thurl et al. [87], to determine the four different HM types (Table 3), represented by the samples HM II-V, 

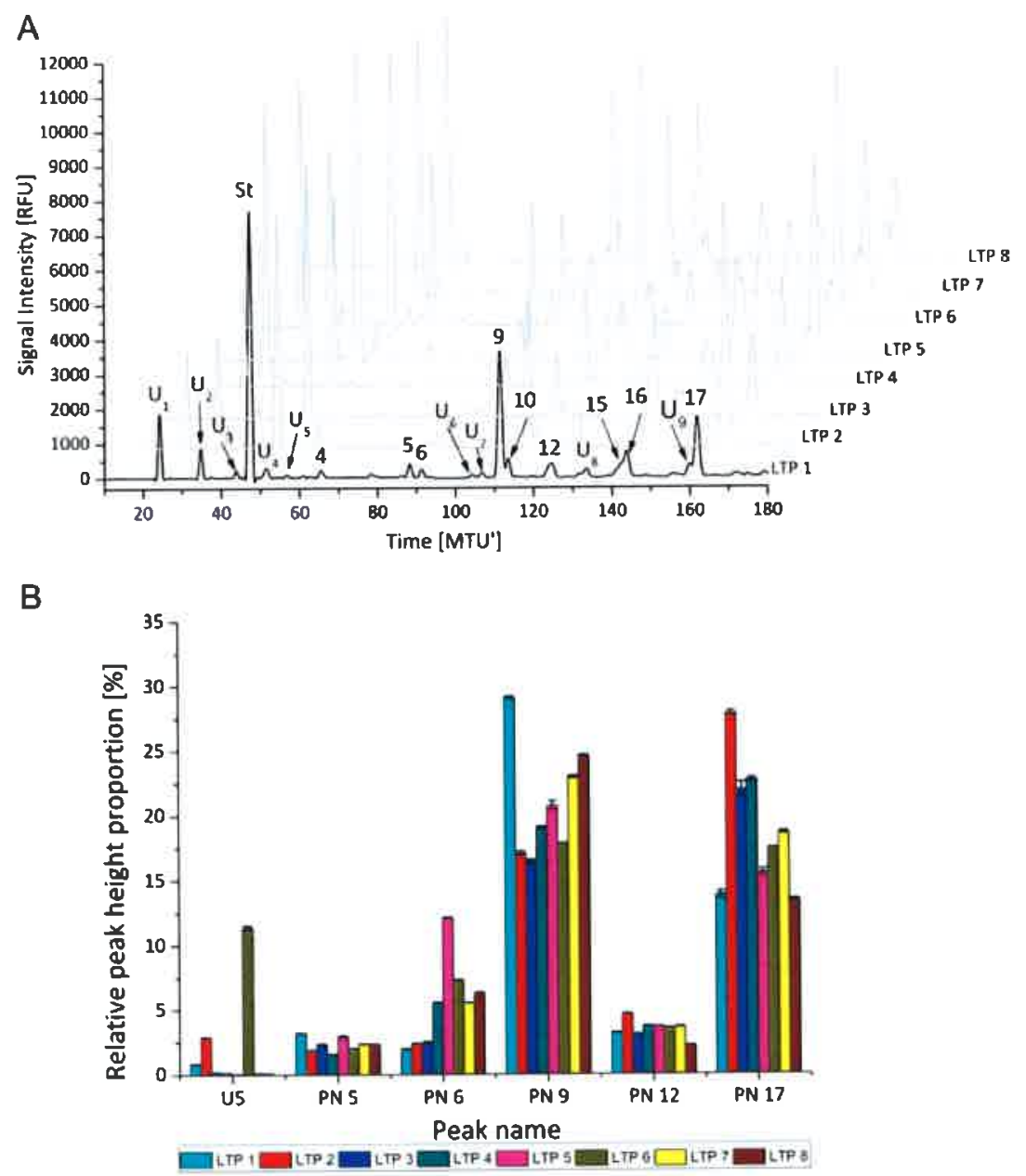

Figure 4. HMOS fingerprints from samples taken along lactation time course and bar plot of the relative PHP of selected peaks. (A) Shifted waterfall overlay of HMOS fingerprints (normalized electropherograms) plotted over [MTU'] of the nHMOS fraction (HM VI/HM type III) from eight LTP (day 1$3,4,8,15,22,30,60$, and 90 postpartum) of one donor, conducted via XCGE-LIF. For each LTP, the same 18 peaks were picked. Peak nomenclature: St: stachyose peak, covering the fucosyllactoses; PN 4-17 (according to Table 2) and $U_{1}-U_{9}$ (unknown peaks). (B) Bar plot of the relative PHP (see Table 4) of selected HMOS peaks $\left(U_{5}, P N 5\right.$, $6,9,12$, and 17) along the lactation time course. The chosen peaks represent relatively constant HMOS (PN 5 and 12), respectively, HMOS significantly changing their concentration along the lactation time course ( $\triangle$ PHP $\gg 10 \%$ of the actual PHP: $U_{5}, P N 6,9$, and 17). [Stachyose was added to each sample during the sample preparation (HM-TCF fractionation via GPC) for subsequent quantification of the HMOS components via HPAECPAD analysis (according to Thurl et al. [26]). During the CGE-LIF based OS analysis, it became clear that stachyose interferes with two HMOS peaks. The migration time of stachyose is nearly the same as for the two fucosylactoses (PN 2 and 3). In Fig. 4, this sum peak is marked with St and is later on not considered in detail, since there is no possibility to distinguish between stachyose, $3-\mathrm{FL}$, and $2^{\prime}-\mathrm{FL}$.]

the presence or absence of distinct HMOS had to be investigated. For doing so, the peaks within the HMOS fingerprints (Fig. 3A and C-E and peak numbers (PN) 1-17) were assigned via normalized migration time matching with the database entries of Table 2. In addition, this HMOS determination was confirmed by consecutive reconducting the fingerprints, after spiking each of the 17 isolated and labeled HMOS standards of Table 2 to each of the four individual HM samples (i.e. $17 \times 4$ additional runs; data not shown). Figure 3A represents the HMOS fingerprint of HM II, in which all peaks (PN 1-17 (Table 2)) appear. Figure 3B shows an additional zoom into this HMOS fingerprint to reveal the ability of the xCGE-LIF based HMOS analysis method to resolve/separate the four isomeric Lacto- $N$-fuco-pentaoses (LNFP I, II, III, and $\mathrm{V}$ (PN 7-10)). As a result (according to Table 3 and Thurl et al. [87]), HM II clearly represents HM type I, which corresponds to secretor gene $(\mathrm{Se})$ as well as Lewis gene ( $L e)$ positive status of the donor.

In Fig. 3C, the peaks PN 3, 4, 9. 13, 16, and 17 do not appear in the HMOS fingerprint of HM III. Thus, according to Table 3 and [87], by the lack of these peaks HM type II is indicated for this donor. In Fig. 3D, LNFP II (PN 7) and additionally, the hexaoses LNDH I (PN 13) and LNDH II (PN 14) are absent, i.e. according to Table 3 and [87], the HMOS fingerprint of HM IV corresponds to the TCF composition of an HM type III. Figure 3E illustrates the HMOS fingerprint of HM V. According to Table 3 and [87], this donor could be identified to be HM type IV, as the peaks PN 3, 4, 7, 9, 13, 14, and 16 do not appear in this HMOS fingerprint. Compared to the HM types I-III (Fig. 3A, C, and D) here the lowest number of peaks was found. In addition, the large proportion of Lacto- $\mathrm{N}$ tetraose (PN G) is striking in Fig. 3E, making Lacto- $N$-tetraose (beside PN 1 (lactose)) to the by far most abundant HMOS within this sample. During the examination of the HMOS fingerprints (Fig. 3A and C-E), significant differences could be identified and based on these differences HM typing was easily possible. For better illustration of the differences between the four HM types, all four fingerprints are plotted as an overlay in Fig. 3F. Via overlay, the excellent separation performance is demonstrated and the striking reproducibility of the developed XCGE-LIF based HMOS analysis method is shown by the coincidence of the peaks within the overlayed 
Table 4. Summary of the relative PHP $(\%)$ of selected peaks along lactation time course of HM VI (see Table 1)

\begin{tabular}{lrrrrrrrr}
\hline Peak PN & LTP 1 & LTP 2 & LTP 3 & LTP 4 & LTP 5 & LTP 6 & LTP 7 & LTP 8 \\
\hline$U_{1}$ & 14.95 & 11.03 & 22.71 & 19.64 & 20.08 & 16.43 & 18.53 & 25.90 \\
$U_{2}$ & 6.82 & 2.72 & 2.40 & 1.76 & 1.65 & 1.43 & 0.70 & 0.65 \\
$U_{3}$ & 1.64 & 0.31 & 0.36 & 0.20 & 0.08 & 0.13 & 0.10 & 0.03 \\
$U_{4}$ & 2.42 & 0.94 & 2.06 & 1.37 & 2.07 & 1.25 & 1.43 & 2.41 \\
$U_{5}$ & 0.82 & 2.89 & 0.18 & 0.09 & 0.03 & 11.37 & 0.09 & 0.05 \\
4 & 1.74 & 0.19 & 1.71 & 0.65 & 1.04 & 0.58 & 0.76 & 0.52 \\
5 & 3.17 & 1.87 & 2.30 & 1.55 & 2.92 & 2.01 & 2.36 & 2.32 \\
6 & 1.94 & 2.37 & 2.45 & 5.54 & 12.11 & 7.27 & 5.52 & 6.35 \\
$U_{6}$ & 0.59 & 0.49 & 0.78 & 0.59 & 0.16 & 0.34 & 0.32 & 0.24 \\
$U_{7}$ & 1.11 & 0.56 & 0.29 & 0.09 & 0.03 & 0.05 & 0.06 & 0.08 \\
9 & 29.10 & 17.06 & 16.42 & 19.07 & $\mathbf{2 0 . 7 6}$ & 17.84 & 22.97 & 24.62 \\
10 & 4.35 & 1.78 & 4.45 & 2.60 & 3.96 & 2.95 & 3.96 & 4.97 \\
12 & 3.17 & 4.65 & 3.06 & 3.69 & $\mathbf{3 . 6 5}$ & 3.56 & 3.63 & 2.22 \\
$U_{8}$ & 1.94 & 0.26 & 0.69 & 0.21 & $\mathbf{0 . 1 8}$ & 0.31 & 0.33 & 0.26 \\
15 & 3.61 & 7.68 & 6.95 & 10.31 & 7.79 & $\mathbf{B . 2 3}$ & $\mathbf{8 . 6 9}$ & 6.40 \\
16 & 6.02 & 15.17 & 6.81 & $\mathbf{6 . 7 6}$ & $\mathbf{5 . 9 3}$ & $\mathbf{5 . 9 4}$ & $\mathbf{6 . 0 1}$ & 3.65 \\
$\mathrm{U}_{\mathbf{g}}$ & 2.77 & 2.19 & 4.42 & 3.14 & 2.01 & 2.90 & 5.91 & 5.88 \\
17 & $\mathbf{1 3 . 8 2}$ & 27.87 & 21.96 & 22.73 & 15.54 & 17.42 & $\mathbf{1 8 . 6 3}$ & 13.43 \\
\hline
\end{tabular}

PN 4-17 (according to Table 2) and $U_{1}-U_{9}$ (unknown peaks). Color code of the comparison of the relative PHP (always against predecessor), black: constant (change of PHP below $10 \%=\mathrm{RSD}_{\max }$ ), red: decrease of PHP $>10 \%$; green: increase of $\mathrm{PHP}>10 \%$.

fingerprints. Second, as mentioned above, samples HM VI of one donor (Table 1) from eight consecutive LTP were analyzed in three technical replicates to prove the feasibility of the method and to demonstrate the feature of relative quantitation for the purpose of monitoring lactation time courses of larger cohorts in the future. For this series of experiments, first, the HM-TCF of each LTP was separated into the nHMOS fraction and the aHMOS fraction (see Section 2). In the following, only the nHMOS fractions were analyzed via xCGELIF, shown in Fig. 4. To visualize the (quantitative) changes along the lactation time course, the HMOS fingerprints of these HM samples are plotted as shifted waterfall overlay (Fig. 4A). Based on the absence of the peaks corresponding for LNFP II (PN 7) and the LNDH I and II (PN 13 and 14) inside the HMOS fingerprint, easily HM type III could be determined for this HM. The concrete numbers of quantitative changes of the nHMOS fraction composition, corresponding to relative changes of single peaks along the lactation time course, are given in Table 4 as relative PHP.

The HMOS fingerprints of the nHMOS fraction of all eight LTP (LTP 1 (day 1-3) in black and LTP 2-8 in gray) are shown for the normalized migration time range of 10 to $180 \mathrm{MTU}^{\prime}$ in Fig. 4A. This timespan was chosen to be shown, because all high abundant HMOS compounds as well as the already identified HMOS migrate within this window. Within all eight examined HMOS fingerprints of the samples taken along the lactation time course, the same 18 peaks were selected for peak assignment and relative quantitation. The database matching resulted in the following findings: unfortunately 3 -FL and $2^{\prime}$-fucosyllactose $\left(2^{\prime}-\mathrm{FL}\right)$ were covered by the stachyose-peak (St) (Fig. 4A), PN 4-17 could be as signed (according to Table 2 ) and the peaks $\mathrm{U}_{1}-\mathrm{U}_{9}$, had to be annotated as unknown. See Table 4, which also summarizes the relative PHP of all 18 picked peaks at all LTP. For the three technical replicates of the samples HM VI from eight LTP, an RSD $\leq 10 \%$ was found for the relative PHP values. As a result, it was found that it is easily possible to monitor quantitatively changes in HM-TCF composition during lactation time course using the developed XCGE-LIF based HMOS analysis method. Interestingly, the qualitative composition of the nHMOS fraction remains constant along the lactation time course (all peaks could be identified at all time points (Fig. 4 and Table 4)), while its relative quantitative com. position changes greatly. Exemplarily, peak $U_{5}$ shows large changes within its relative PHP: there is a threefold change from nearly $1 \%$ at the LTP 1 to almost 3\% at LTP 2 and a drop-down to about $1 / 15$ of this value afterwards (Table 4 and Fig. 4B). In the further lactation time course, the relative PHP stays on this "very" low level, except for the LTP 6 (day 30 postpartum). Here the relative PHP of $U_{5}$ reaches by far the biggest value ( $>11 \%$ ), and $U_{5}$ is the fourth abundant peak within the HMOS fingerprint. Another example showing major changes is PN 9 (LNFP I). During the lactation time course, its relative PHP decreases from $29 \%$ at LTP 1 to a minimum of $16.4 \%$ at LTP 3 (Table 4 and Fig. 4B). In contrast to these strongly changing peaks, there are also peaks like PN 5 or 12 that show a quite stable relative PHP during lactation period (Table 4 and Fig. 4B).

\section{Discussion}

The aim of the present study was the establishment of a fast, robust, reliable, and reproducible (i.e. high-throughput), but also sensitive, selective, and highly resolving (i.e. highperformance) HMOS analysis method. It should be shown that the developed xCGE-LIF based method allows generation of HMOS fingerprints to be used for HM typing and can be used for in-depth analysis of HMOS, i.e. structural elucidation of the single compounds and their relative quantification. Therefore first, neat HMOS compounds were isolated out of the HM-TCF (see Section 2, Fig. 2) and were used for method development as well as robustness/reproducibility testing. Second, these neat HMOS were used to initiate a respective database (HMOS structures with their associated $t_{\text {mig }}$ ) for fast and easy identification of HMOS beyond peaks of generated HMOS fingerprints from real samples via migration time matching. Finally, for method establishment, the HM-TCF of four different donors, representing the four known different HM types (see Table 1 and Thurl et al. [87]), as well as a set of eight HM samples of one donor collected at different time-points along the lactation time course, were analyzed repeatedly. Thus, the method could be approved to be a valuable tool for qualitative and quantitative monitoring HMOS pool composition of large HM donor cohorts in future. 


\subsection{Method development - reproducibility testing and initialization of an XCGE-LIF based HMOS database and library}

The successful reproducibility study was prerequisite for the unambiguous assignment of HMOS structures to normalized xCGE-LIF migration times. Reproducibility is the basis for the establishment of an HMOS database that com. bines structural and $\mathbf{t}_{\text {nig }}$ information for each HMOS compound entry. For the future, this database has to be increased to facilitate fast and efficient analysis of the HM-TCF, but also of other OS samples, e.g. animal milk $[104,105]$ or plants as sources of dietetic OS. As already demonstrated for the human blood serum glycome [93], a respective database will also allow the HT analysis of biotechnological HMOS [106] or for HT glycoprofiling of bacterial HMOS consumption [107].

The decisive reproducibility test (technical replicates and repeated measurements along a timespan of 6 months, with a total $N \geq 10$ per HMOS) was passed successfully. The achieved mean $\mathrm{t}_{\text {mig' }}$ and RSD of all 17 repeatedly measured neutral HMOS can be found in Table 2. During this long-term study, two aspects became apparent and should be noted: older capillary arrays lose sensitivity and overaged polymer hampers the overall stability of the system. To avoid these phenomena, both the capillary array and the polymer were changed regularly. As the temperature has an effect on the viscosity of the polymer, the observed slight fluctuations in $t_{\text {mig }}$ ' could be further reduced by air conditioning.

Having a closer look on the four isomeric fucopentaoses (LNFP I, II, III, and V), it is obvious that their structure has a significant influence on $t_{\text {mig }}{ }^{\prime}$. Their fucose-position ( $\alpha 1-2, \alpha 1-3$, or $\alpha 1-4)$ combined with their core structure has a significant effect on their migration behavior (Fig. 3B and Table 2). Despite identical molecular weights, a $t_{\text {mig }}{ }^{\prime}$ difference of about $5 \mathrm{MTU}^{\prime}$ can be observed between LNFP II and LNFP III. This can be explained by the two orthogonal separation mechanisms working together in CGE: separation by $m / z$ and separation by molecular shape, i.e. all LNFP isomers have the same $m / z$ (three negative charges from APTS and identical molecular weights) but show different glycosidic linkages between their constituent monosaccharides and therefore different molecular shapes. At present, only broad rules with respect to the migration order can be applied (e.g. (i) larger (HM)OS show longer $t_{\text {nigig'; }}$ (ii) negative charges (like additional sialic acid) reduce $t_{\text {mig }}$; (iii) nonfucosylated (HM)OS with Galß1-3 $N$-acetylglucosamine (GlcNAc) core structure (type 1 core) migrate slower than corresponding (HM)OS with Gal $\beta 1-4$ GlcNAc neo-core-structure (type 2 core); and (iv) fucosylated (HM)OS with a Galß1-3GlcNAc core structure migrate faster than corresponding (HM)OS with Gal $\beta 1$ 4GlcNAc (neo)-core structure). According to Rohrer [108], who already summarized regularities for the HPAEC-PADbased (HM)OS analysis, in future the increasing number of HMOS database entries and the increasing knowledge regarding structure-specific CGE separation charac- teristics will allow further preclassification or maybe even prediction of unknown (HM)OS structures within those fingerprints.

As a result of the performed long-term reproducibility testing, it can be stated that the $t_{\text {mig }}$ are quite stable. Over the entire reproducibility study, the RSD of $\mathrm{t}_{\text {mig }}{ }^{\prime}$ was $<1 \%$. for all 17 isolated and tested HMOS. This result indicates that the developed HT and HP xCGE-LIF based HMOS analysis method is appropriate to initialize a respective HMOS database, as its migration time variance is significantly below its typical peak width. The database could now be tested regarding its feasibility to be used for structural elucidation of peaks in real samples.

\subsection{Method application-approval of XCGE-LIF based method regarding qualitative and quantitative analysis of real HMOS samples}

After successful approval of the methods reproducibility and the initialization of a respective HMOS database and library, HMOS fingerprints of HM-TCF of different donors were conducted via xCGE-LIF to demonstrate its performance and applicability. This was successfully demonstrated for HM from donors representing the four known different $\mathrm{HM}$ types. as described in Thurl et al. [87] (Fig. 3) and also for HM samples of one donor from eight different LTP along the lactation time course, as described in [27] (Fig. 4).

Figure $3 \mathrm{~A}$ and $\mathrm{C}-\mathrm{E}$ show the HMOS fingerprints of the four HM donors with HMOS structures assigned to the peaks. Despite the large concentration differences, structures could be easily assigned via database matching (Table 2), e.g. all four isomeric LNFP structures (see zoom-in, Fig. 3B). The assignment of all entries was additionally confirmed by spiking experiments with the corresponding isolated neat HMOS compounds (data not shown). The fingerprints of HM-TCF in Fig. 3 reveal that XCGE-LIF based HMOS analysis nicely resolves very complex HMOS mixtures. This complexity is due to the possibility of different glycosidic linkages that enable a low number of different monosaccharides to form out a broad variety of HMOS structures $[5,28,109-111]$. At this point, it should be noted that some of the various HMOS structures that exist may elute closely together or even may co-elute (e.g. the hexoses LNH (PN 12) and LNnH (PN 11) which are only $0.5 \mathrm{MTU}^{\prime}$ apart, or the stachyose peak which is co-eluting with 3-FL (PN 2) and 2'-FL (PN 3) (Fig. 4). But the majority of these HMOS (including isomeric structures like LNFP (Fig. 3B)) can be clearly separated from each other. Due to the high resolution of the XCGE-LIF based HMOS analysis, all four HM types could easily be determined, according to Thurl et al. [87]. The (HM)OS that contain antigenic determinants of the $L e$ and/or $S e[37,41,112-114]$ are synthesized by fucosyltransferases that are not distributed uniformly throughout the human population (Table 3 ) $[28,115-120]$. The two mentioned antigenic determinants ( $S e$ and $L e$ ) are responsible for the presence of specific fucosyltransferases (FucT-II and III). A secretor $(\mathrm{Se}+)$ bears FucT-II and will build up 
(HM)OS-epitopes, such as $2^{\prime}$-FL, which carry a fucosylation in $\alpha 1-2$ position on the terminal galactose and a nonsecretors will not assemble such (HM)OS-epitopes (Table 3). Le encodes FucT-III, which catalyzes the addition of fucose in $\alpha 1-3$ and $\alpha 1-4$ position on the subterminal GlcNAc. Experiments showed that other enzymes can compensate the $\alpha 1-3$ fucosylation at GlcNAc [27], but apparently only FucT-[II is able to fucosylate GlcNAc in an $\alpha 1-4$ position. Le-dependent OS are normally found as components of glycosphingolipids and glycoproteins in red blood cells and other tissues and biological fluids such as saliva and plasma [121]. However, HM contains these OS in a free form. Based on the presence or absence of these specific HMOS, it is possible to classify HM into specific groups. Table 3 lists and classifies the possible glycosidic linkages (fucosylation) based on $\mathrm{Se}$ and Le. Donors classified with HM type I are $S e$ and $L e$ positive and thus hold the genetic requirements for a "complete" HMOS equipment. Donors classified with HM type IV are $S e$ as well as $L e$ negative. Consequently, they do not have FucT-II and III and do not build up the respective fucosylated HMOS structures, i.e. HMOS with $\alpha 1-2$ fucosysated terminal galactose or a $\alpha 1.4$ fucosylated GlcNAc cannot be synthesized by donors with HM type IV. Accordingly, HMOS structures like $2^{\prime}$-FL (PN 3), LNFP II (PN 7), LNFP I (PN 9), LNDH I (PN 13), LNDH II (PN 14), 2'-fucosyl-Lacto- $N$-hexaose (PN 16). and 2,3-difucosyl-Lacto- $\mathrm{N}$-hexaose (PN 17) are missing for HM type IV (Table 2 and Fig. 3E). Absence of the respective fucosylated HMOS structures could also be revealed within the HMOS fingerprints of HM type II (Fig. 3C) and III (Fig. 3D), while all expected fucosylated HMOS were found within the HM type I fingerprint (Fig. 3A).

Furthermore, the high concentration of lactose (PN 1) in all four HMOS fingerprints (that can be explained by the extremely high lactose concentration from 53-61 g/L within the HM-TCF [122]) should be noted: by far this signal exceeds the linear dynamic range (above $9000 \mathrm{RFU}$ ) of the detector and consequently the lactose peak tip appears splitted as can be seen in Fig. 3C and D (PN 1). But, in contrast to chromatographic methods, for electrophoretic techniques there is no effect whether lactose is within the sample at high concentrations or not. Almost no peak broadening in xCGE-LIF can be observed for the lactose peak compared to what has to be expected for chromatographic techniques. When using LC techniques for HMOS profiling, the lactose content of each sample has to be reduced $[26,27,87,108]$, to avoid column overloading leading to peak broadening and disturbed chromatograms. This superior loading capacity of electrophoretic techniques, like XCGE-LIF, is due to the fact, that electrophoresis is a volume-based separation technique, while adsorption/dispersion chromatography is surface based and thus provides only limited area for the adsorption/dispersion process. Another striking advantage of XCGE-LIF based HMOS analysis is the possibility to simultaneously analyze acidic and neutral HMOS, which can be allocated to its superior separation performance.

The comparative investigation of the TCF of different HM types showed, that not only the qualitative HMOS pool composition differs, but also the relative abundance of specific HMOS can do so. The possibility to measure such quantitative changes within the HM-TCF composition is shown to be indispensable with respect to monitoring along the lactation time course $[9,28,32]$. Therefore as the final part of this initial work, HM samples from eight different LTP of one donor (HM VI, see Table 1) were analyzed to demonstrate the feasibility of this method to monitor changes within the HMOS composition during the lactation time course. The analyzed time points are summarized in Table 1. To confirm the results of the xCGE-LIF based HMOS analysis, the samples were additionally analyzed using HPAEC-PAD according to $[27,87]$. HPAEC-PAD was used to validate the xCGE-LIF results, as this orthogonal method is the most accepted reference technique in the field of (HM)OS analysis (data not shown). As HPAEC-PAD is also routinely applied to quantify HMOS, a defined amount of stachyose had been already added to these samples before we started sample preparation (see Section 2). Stachyose is a tetraose and within an HPAEC-PAD chromatogram and there, shows no impact to HMOS peaks (data not shown). However, for the xCGE-LIF based HMOS analysis of these eight samples, it became evident that stachyose and the fucosyllactoses (PN 2 and 3) show almost identical migration times (about 47 $\left.\mathrm{MTU}^{\prime}\right)$. Thus, the sum peak of stachyose, 3-FL, and $2^{\prime}-\mathrm{FL}$ as shown in Fig. 4A (marked with St) was not further considered. As depicted in Fig. 4 and listed in Table 4, the individual changes within the HMOS pool composition along the lactation time course could easily be monitored. The relative PHP of 18 specific peaks over the lactation period are summarized in Table 4. Looking at this table, it is obvious that some of the HMOS change quite strongly in their relative PHP. Large quantitative changes can be seen, e.g. for PN 6, 9, 17 and in particular, for $\mathrm{U}_{5}$ that showed by far the biggest changes in its relative PHP (Fig. 4B). But there are also peaks remaining relatively constant (e.g. PN 5 and 12 - Table 4 and Fig. 4B), over the lactation period. At this time point, an explanation of these changes has to wait for further investigations, as up to now only few attempts were made to understand the quantitative changes of HMOS within (human) milk throughout the lactation time course and also regarding the elucidation of their functions. Despite the known importance of complex carbohydrates (like $\mathrm{N}$-glycans, $\mathrm{O}$-glycans, and free $\mathrm{OS}$ ) in (human) milk $[2,20,77,83,123-129]$, only a few publications are concerning this topic and were analyzing larger donor cohorts $[22,27-32,39]$ therefore. One reason is probable, that up to now, there was no "real" HT analysis method available, that allows the measurement of thousands of samples within a couple of days $[83,130]$.

Next task will be to enhance the initiated HMOS database (starting with structural elucidation of the unknown peaks $\left.\left(U_{1}-U_{9}\right)\right)$, to reach a sufficient number of entries. In combination the presented sample preparation, the HMOS database, the software glyXtool ${ }^{T M}$ [97], and the possibility to use instruments with up to 96 capillaries in parallel will empower the developed xCGE-LIF based method for HMOS analysis to conduct parallel measurements with an effective runtime 
$<1$ min for each sample [131]. This HP and "real" HT glycoanalytical technique will enable large-scale studies, which will allow further insights into functional glycomics of free HMOS, digging deeper into the relation of mothers genotype, HMOS pool composition, and HM properties/effects as lately requested from Neville et al. [2].

The authors would like to thank Sabine jelinek for the excellent technical support. This work has been supported with funding from the European Union's Seventh Framework Programme (FP7-Health-F5-2011) under grant agreement number 278535 (HighGlycan).

The authors have declared no conflict of interest.

\section{References}

[1] World Health Organisation 2012, 55th World Health Assembly, A55/15, Infant and young child nutrition: Global strategy on infant and young child feeding. http://apps.who.int/gb/archive/pdf_files/ WHA55/ea5515.pdf.

[2] Neville, M. C., Anderson, S. M., McManaman, J. L., Badger, T. M., Bunik, M., Contractor, N., Crume, T., Dabelea, D., Donovan, S. M., Forman, N., Frank, D. N., Friedman, J. E., German, J. B., Goldman, A., Hadsell, D., Hambidge, M., Hinde, K., Horseman, N. D., Hovey, R. C., Janoff, E., Krebs, N. F., Lebrilla, C. B., Lemay, D. G., Maclean, P. S., Meier, P., Morrow, A. L., Neu, J., Nommsen-Rivers, L. A., Raiten, D. J., Rijnkels, M., Seewaldt, V., Shur, B. D., Vanhouten, J., Williamson, P., J. Mammary Gland Biol. 2012, 17, 167-188.

[3] Emmett, P. M., Rogers, I. S., Early Hum. Dev. 1997, 49 (Suppl), S7-S28.

[4] Rodriguez-Palmero, M., Koletzko, B., Kunz, C., Jensen, R., Clin. Perinatol. 1999, 26, 335-359.

[5] Kunz, C., Rudloff, S., Baier, W., Klein, N., Strobel, S., Annu. Rev. Nutr. 2000, 20, 699-722.

[6] Kunz, C., Rodriguez-Palmero, M., Koletzko, B., Jensen, R., Clin. Perinatol. 1999, 26, 307-333.

[7] Blanc, B., World Rev. Nutr. Diet 1981, 36, 1-89.

[8] Ballard, O., Morrow, A. L., Pediatr. Clin. North America 2013, 60, 49-74.

[9] Prentice, A., Food Nutr. Bull. 1996, 17, 305-312.

[10] Wells, J., Nursing Times 1986, 82, 53-55.

[11] Van de Perre, P., Vaccine 2003, 21, 3374-3376.

[12] Glass, R. I., Svennerholm, A. M., Stoll, B. J., Khan, M. R., Hossain, K. M., Huq, M. I., Holmgren, J., N. Engl. J. Med. 1983, 308, 1389-1392.

[13] Chichlowski, M., German, J. B., Lebrilla, C. B., Mills, D. A., Annu. Rev. Food Sci. Technol. 2011, 2. 331-351.

[14] Bode, L., Glycobiology 2012, 22, 1147-1162.

[15] Hanson, L. A., Ahlstedt, S., Andersson, B., Carlsson, B., Fallstrom, S. P., Mellander, L., Porras, O., Soderstrom, T., Eden, C. S., Pediatrics 1985, 75, 172-176.

[16] Hanson, L. A., Ann. Allerg. Asthma Immunol. 1998, 81, 523-533.
[17] Schack-Nielsen, L., Michaelsen, K. F., Curr. Opin. Clin. Nutr. Metab. Care 2006, 9, 289-296.

[18] Dewey, K. G., Heinig, M. J., Nommsen-Rivers, L. A., J. Pediatr. 1995, 126, 696-702.

[19] Marild, S., Hansson, S., Jodal, U., Oden, A., Svedberg, K., Acta Paediatr. 2004, 93, 164-168.

[20] Bode, L., Nutr. Rev. 2009, 67 (Suppl 2), S183-S191.

[21] Saarela, T., Kokkonen, J., Koivisto, M., Acta Paediatr. 2005, 94, 1176-1181.

[22] Yu, Y., Mishra, S., Song, X., Lasanajak, Y., Bradley, K. C., Tappert, M. M., Air, G. M., Steinhauer, D. A., Halder, S., Cotmore, S., Tattersall, P., AgbandjeMcKenna, M., Cummings, R. D., Smith, D. F., J. Biol. Chem. 2012, 287, 44784-44799.

[23] Blank, D., Gebhardt, S., Maass, K., Lochnit, G., Dotz, V., Blank, J., Geyer, R., Kunz, C., Anal. Bioanal. Chem. $2011,401,2495-2510$.

[24] Blank, D., Dotz, V., Geyer, R., Kunz, C., Adv Nutr. 2012, 3, 440S-449S.

[25] Marino, K., Lane, J. A., Abrahams, J. L., Struwe, W. B., Harvey, D. J., Marotta, M., Hickey, R. M., Rudd, P. M., Glycobiology 2011, 21, 1317-1330.

[26] Thurl, S., MullerWerner, B., Sawatzki, G., Anal. Biochem. 1996, 235, 202-206.

[27] Thurl, S., Munzert, M., Henker, J., Boehm, G., MullerWerner, B., Jelinek, J., Stahl, B., Br. J. Nutr. 2010, 104, 1261-1271.

[28] Erney, R. M., Malone, W. T., Skelding, M. B., Marcon, A. A., Kleman-Leyer, K. M., O'Ryan, M. L., Ruiz-Palacios, G., Hilty, M. D., Pickering, L. K., Prieto, P. A., J. Pediatr. Gastroenterol. Nutr. 2000, 30, 181-192.

[29] Erney, R., Hilty, M., Pickering, L., Ruiz-Palacios, G., Prieto, P., Adv. Exp. Med. Biol. 2001, 501 , 285-297.

[30] Newburg, D. S., Warren, C. D., Chaturvedi, P., Ruiz Palacios, G., Pickering, L. K., Faseb J. 1997, 11, 1385-1385.

[31] Coppa, G. V., Gabrielli, O., Pierani, P., Catassi, C., Carlucci, A., Giorgi, P. L., Pediatrics 1993, 91, 637-641.

[32] Ninonuevo, M. R., Perkins, P. D., Francis, J., Lamotte L. M., LoCascio, R. G., Freeman, S. L., Mills, D. A., German, J. B., Grimm, R., Lebrilla, C. B., J. Agr. Food Chem. 2008, 56, 4854-4854.

[33] Ninonuevo, M. R., Park, Y., Yin, H., Zhang, J., Ward, R. E., Clowers, B. H., German, J. B., Freeman, S. L. Killeen, K., Grimm, R., Lebrilla, C. B., J. Agric. Food Chem. 2006, 54, 7471-7480.

[34] Asakuma, S., Urashima, T., Akahori, M., Obayashi, H., Nakamura, T., Kimura, K., Watanabe, Y., Arai, I., Sanai, Y., Eur. J. Clin. Nutr. 2008, 62, 488-494.

[35] Coppa, G. V., Gabrielli, O., Zampini, L., Galeazzi, T., Ficcadenti, A., Padella, L., Santoro, L., Soldi, S., CarIucci, A., Bertino, E., Morelli, L., J. Pediatr. Gastr. Nutr. 2011, 53, 80-87.

[36] Viverge, D., Grimmonprez, L., Cassanas, G., Bardet, L., Solere, M., J. Pediatr. Gastr. Nutr. 1990, 11, 361-364.

[37] Viverge, D., Grimmonprez, L., Cassanas, G., Bardet, L., Solere, M., J. Pediatr. Gastr. Nutr. 1990, 11, 365-370. 
[38] Coppa, G. V., Pierani, P., Zampini, L., Carloni, I., Carlucci, A., Gabrielli, O., Acta Paediatr. Suppl. 1999, 88, 89-94.

[39] Totten, S. M., Zivkovic, A. M., Wu, S., Ngyuen, U., Freeman, S. L., Ruhaak, L. R., Darboe, M. K., German, J. B., Prentice, A. M., Lebrilla, C. B., J. Proteome Res. 2012, 11, 6124-6133.

[40] De Leoz, M. L., Gaerlan, S. C., Strum, J. S., Dimapasoc, L. M., Mirmiran, M., Tancredi, D. J., Smilowitz, J. T., Kalanetra, K. M., Mills, D. A., German, J. B., Lebrilla, C. B., Underwood, M. A., J. Proteome Res. 2012, 11, 4662-4672.

[41] Kobata, A., in: Colowick, S., Kaplan, N. (Eds.), Methods in Enzymology Menza, New York 1972, pp. 262-271.

[42] Kobata, A., Ginsburg, V., Tsuda, M., Arch. Biochem. Biophys. 1969, 130, 509-513.

[43] Kobata, A., Ginsburg, V., J. Biol. Chem. 1969, 244, 54965502.

[44] Kobata, A., Ginsburg, V., Arch Biochem Biophys 1972, $150,273-281$

[45] Kobata, A., Ginsburg, V., Journal of Biological Chemistry 1972, 247, 1525-1529.

[46] Chaturvedi, P., Warren, C. D., RuizPalacios, G. M., Pickering, L. K., Newburg, D. S., Anal. Biochem. 1997, 251. 89-97.

[47] Guile, G. R., Rudd, P. M., Wing, D. R., Prime, S. B., Dwek, R. A., Anal. Biochem. 1996, 240, 210-226.

[48] Kobata, A., Proc. Jpn. Acad. Ser. B, Phys. Biol. Sci. 2010, $86,731-747$.

[49] Wang, W. T., Erlansson, K., Lindh, F, Lundgren, T., Zopf, D., Anal. Biochem. 1990, 190, 182-187.

[50] Wang, W. T., Zopf, D., Carbohydr. Res. 1989, 189, 1-11.

[51] Hardy, M. R., Townsend, R. R., Carbohydr. Res. 1989, $188,1-7$.

[52] Townsend, R. R., Hardy, M., Olechno, J. D., Carter, S. R., Nature 1988, 335, 379-380.

[53] Townsend, R. R., Hardy, M. R., Lee, Y. C., Methods Enzymol. 1989, 179, 65-76.

[54] Reddy, G. P., Bush, C. A., Anal. Biochem. 1991, 198, 278-284.

[55] Zauner, G., Deelder, A. M., Wuhrer, M., Electrophoresis $2011,32,3456-3466$.

[56] Ninonuevo, M. R., Lebrilla, C. B., Nutr. Rev. 2009, 67 (Suppl 2), S216-S226.

[57] Spengler, B., Dolce, J. W., Cotter, R. J., Anal. Chem. 1990, 62, 1731-1737.

[58] Hofmeister, G. E., Zhou, Z., Leary, J. A., J. Am. Chem. Soc. 1991, 113, 5964-5970.

[59] Stahl, B., Steup, M., Karas, M., Hillenkamp, F., Anal. Chem. 1991, 63, 1463-1466.

[60] Stahl, B., Thurl, S., Zeng, J., Karas, M., Hillenkamp, F., Steup, M., Sawatzki, G., Anal. Biochem. 1994, 223, 218-226.

[61] Finke, B., Stahl, B., Pfenninger, A., Karas, M., Daniel, H., Sawatzki, G., Anal. Chem. 1999, 71, 3755-3762.

[62] Thibeault, D., Caron, N., Djiana, R., Kremer, R., Blank, D., J. Chromatogr. B Analyt. Technol. Biomed. Life Sci. $2012,883-884,120-127$.
[63] Blank, D., Geyer, H., Maass, K., Yamashita, K., Geyer, R., Anal. Biochem. 2012, 421, 680-690.

[64] Amano, J., Sugahara, D., Osumi, K., Tanaka, K., Glycobiology 2009, 19, 592-600.

[65] Amano, J., Osanai, M., Orita, T., Sugahara, D., Osumi, K., Glycobiology 2009, 19, 601-614.

[66] Pfenninger, A., Karas, M., Finke, B., Stahl, B., J. Am. Soc. Mass Spectrom. 2002, 13, 1341-1348.

[67] Garozzo, D., Impallomeni, G., Spina, E., Green, B. N., Hutton, T., Carbohydr. Res. 1991, 221, 253-257.

[68] Mulroney, B., Traeger, J. C., Stone, B. A., J. Mass Spectrom. 1995, 30, 1277-1283.

[69] Pfenninger, A., Karas, M., Finke, B., Stahl, B., J. Am. Soc. Mass Spectrom. 2002, 13, 1331-1340.

[70] Park, Y., Lebrilla, C. B., Mass Spectrom. Rev. 2005, 24, 232-264.

[71] Fong, B., Ma, K., McJarrow, P., J. Agric. Food Chem. 2011, 59, 9788-9795.

[72] Strum, J. S., Aldredge, D., Barile, D., Lebrilla, C. B., Anal. Biochem. 2012, 424, 87-96.

[73] Galeotti, F., Coppa, G. V., Zampini, L., Maccari, F., Galeazzi, T., Padella, L., Santoro, L., Gabrielli, O., Volpi, N., Anal. Biochem. 2012, 430, 97-104.

[74] Thomsson, K. A., Schulz, B. L., Packer, N. H., Karlsson, N. G., Glycobiology 2005, 15, 791-804.

[75] Karlsson, N. G., Wilson, N. L., Wirth, H. J., Dawes, P., Joshi, H., Packer, N. H., Rapid Commun. Mass Spectrom. 2004, 18, 2282-2292.

[76] Karlsson, N. G., Schulz, B. L., Packer, N. H., J. Am. Soc. Mass Spectrom. 2004, 15, 659-672.

[77] Bao, Y., Chen, C., Newburg, D. S., Anal. Biochem. 2013, 433, 28-35.

[78] Broberg, A., Carbohydr. Res. 2007, 342, 1462-1469.

[79] Wu, S., Salcedo, J., Tang, N., Waddell, K., Grimm, R., German, J. B., Lebrilla, C. B., Anal. Chem. 2012, 84, 7456-7462.

[80] Haeuw-Fievre, S., Wieruszeski, J. M., Plancke, Y., Michalski, J. C., Montreuil, J., Strecker, G., Eur. J. Biochem. 1993, 215, 361-371.

[81] Fievre, S., Wieruszeski, J. M., Michalski, J. C., Lemoine, J., Montreuil, J., Strecker, G., Biochem. Biophys. Res. Commun. 1991, 177, 720-725.

[82] Bao, Y., Zhu, L., Newburg, D. S., Anal. Biochem. 2007, 370, 206-214.

[83] Bao, Y., Newburg, D. S., Electrophoresis 2008, 29, 2508-2515.

[84] Albrecht, S., Schols, H. A., van den Heuvel, E. G., Voragen, A. G., Gruppen, H., Electrophoresis 2010, 31 , 1264-1273.

[85] Albrecht, S., Schols, H. A., van Zoeren, D., van Lingen, R. A., Groot Jebbink, L. J., van den Heuvel, E. G., Voragen, A. G., Gruppen, H., Carbohydr. Res. 2011, 346, 2540-2550.

[86] Albrecht, S., Schols, H. A., van den Heuvel, E. G., Voragen, A. G., Gruppen, H., Carbohydr. Res. 2011, 346, 2540-2550.

[87] Thurl, S., Henker, J., Siegel, M., Tovar, K., Sawatzki, G., Glycoconj. J. 1997, 14, 795-799. 
[88] Thurl, S., Offermanns, J., Mullerwerner, B., Sawatzki, G., J. Chromatogr. Biomed. 1991, 568, 291-300.

[89] Ruhaak, L. R., Zauner, G., Huhn, C., Bruggink, C., Deelder, A. M., Wuhrer, M., Anal. Bioanal. Chem. 2010, 397, 3457-3481.

[90] Lamari, F. N., Kuhn, R., Karamanos, N. K., J. Chromatogr. B 2003, 793, 15-36.

[91] Harvey, D. J., J. Chromatogr. B 2011, 879, 1196-1225.

[92] Schwarzer, J., Rapp, E., Reichl, U., Electrophoresis 2008 , $29,4203-4214$.

[93] Ruhaak, L. R., Hennig, R., Huhn, C., Borowiak, M. Dolhain, R. J., Deelder, A. M., Rapp, E., Wuhrer, M. J. Proteome Res. 2010, 9, 6655-6664.

[94] Rapp, E., Hennig, R., Borowiak, M., Kottler, R., Reichl U., Glycoconjugate J. 2011, 28, 234-235.

[95] Karas, M., Ehring, H., Nordhoff, E., Stahl, B., Strupat, K., Hillenkamp, F., Grehl, M., Krebs, B., Org. Mass Spectrom. 1993, 28, 1476-1481.

[96] Callewaert, N., Geysens, S., Molemans, F., Contreras, R., Glycobiology 2001, 11, 275-281.

[97] Hennig, R., Reichl, U., Rapp, E., Glycoconjugate J. 2011 , 28,331 .

[98] Schwarzer, J., Rapp, E., Hennig, R., Genzel, Y., Jordan, I., Sandig, V., Reichl, U., Vaccine 2009, 27, 4325-4336.

[99] Ruhaak, L. R., Hennig, R., Huhn, C., Borowiak, M., Dolhain, R. J. E. M., Deelder, A. M., Rapp, E., Wuhrer, M., J. Proteome Res. 2010, 9, 6655-6664.

[100] Roedig, J. V., Rapp, E., Hoper, D., Genzel, Y., Reichl, U., PLoS One 2011, 6, e27989.

[101] Roedig, J. V., Rapp, E., Genzel, Y., Reichl, U., BMC Proc. 2011, 5 (Suppl 8), P113.

[102] Rodig, J. V., Rapp, E., Bohne, J., Kampe, M., Kaffka, H., Bock, A., Genzel, Y., Reichl, U., Biotechnol. Bioeng. 2013, $110,1691-1703$.

[103] Rodig, J., Rapp, E., Djeljadini, S., Lohr, V., Genzel, Y., Jordan, I., Sandig, V., Reichl, U., J. Carbohyd. Chem. 2011, 30, 281-290.

[104] Zivkovic, A. M., Barile, D., Adv. Nutr. 2011, 2, 284-289.

[105] Barile, D., Tao, N., Lebrilla, C. B., Coisson, J. D., Arlorio, M., German, J. B., Int. Dairy J. 2009, 19, 524-530.

[106] Han, N. S., Kim, T. J., Park, Y. C., Kim, J., Seo, J. H., Biotechnol. Adv. 2012, 30, 1268-1278.

[107] Locascio, R. G., Ninonuevo, M. R., Kronewitter, S. R., Freeman, S. L., German, J. B., Lebrilla, C. B., Mills, D. A., Microbial Biotechnol. 2009, 2, 333-342.

[108] Rohrer, J. S., Glycobiology 1995, 5, 359-360.

[109] Wu, S., Tao, N., German, J. B., Grimm, R., Lebrilla, C. B., J. Proteome Res. 2010, 9, 4138-4151.
[110] Wu, S., Grimm, R., German, J. B., Lebrilla, C. B., J. Proteome Res. 2011, 10, 856-868.

[111] German, J. B., Freeman, S. L., Lebrilla, C. B., Mills, D. A., Nestle Nutr. Inst. Workshop Ser. Paediat. Progr. 2008, 62.

[112] Sabharwal, H., Nilsson, B., Chester, M. A., Sjoblad, S., Lundblad, A., Mol. Immunol. 1984, 21, 1105-1112.

[113] Ginsburg, V., McGinniss, M. H., Zopf, D. A., Prog. Clin. Biol. Res. 1980, 43, 45-53.

[114] Ginsburg, V., Adv. Enzymol. Relat. Areas Mol. Biol. $1972,36,131-149$.

[115] Bjarnaso, O., Bjarnaso, V., Edwards, J. H., Fridriks, S., Magnusso, M., Mourant, A. E., Tills, D., Ann. Hum. Genet. 1973, 36, 425-459.

[116] Boettcher, B., Kenny, R., Hum. Hered. 1971, 21, 334-345.

[117] Booth, P. B., Saave, J. J., Hornabrook, R. W., Hum. Biol. Oceania 1973, 2, 155-166.

[118] Molthan, L., Vox Sang. 1980, 39, 327-330.

[119] Ssebabi, E. C., CRC Crit. Rev. Clin. Lab. Sci. 1975, 6, $19-45$.

[120] Welch, S. G., Barry, J. V., Dodd, B. E., Griffith, P. D., Huntsman, R. G., Jenkins, G. C., Lincoln, P. J., Mccathie, M., Mears, G. W., Parr, C. W., Hum. Hered. 1973, 23, 230-240.

[121] Green, C., FEMS Microbiol. Immunol. 1989, 1, 321-330.

[122] Newburg, D. S., Linhardt, R. J., Ampofo, S. A., Yolken, R. H., J. Nutr. 1995, 125, 419-424.

[123] Ruhaak, L. R., Lebrilla, C. B., BMB Reports 2012, 45, $442-451$.

[124] Gnoth, M. J., Kunz, C., Kinne-Saffran, E., Rudloff, S., J. Nutr. 2000, 130, 3014-3020.

[125] Kunz, C., Rudloff, S., Faseb J. 1997, 11, 1386-1386.

[126] Bode, L., J. Nutr. 2006, 136, 2127-2130.

[127] Bode, L., Jantscher-Krenn, E., Adv. Nutr. 2012, 3, 383S-391S.

[128] Gibson, G. R., Roberfroid, M. B., J. Nutr. 1995, 125 , 1401-1412.

[129] German, J. B., Dillard, C. J., Ward, R. E., Curr. Opin. Clin. Nutr. Metab. Care 2002, 5, 653-658.

[130] Ruhaak, L. R., Lebrilla, C. B., Adv. Nutr. 2012, 3, 406S-414S.

[131] Hennig, R., Borowiak, M., Ruhaak, L, R., Wuhrer, M., Rapp, E., Glycoconjugate J. 2011, 28, 331-332.

[132] Ceroni, A., Maass, K., Geyer, H., Geyer, R., Dell, A., Haslam, S. M., J. Proteome Res. 2008, 7, 1650-1659. 